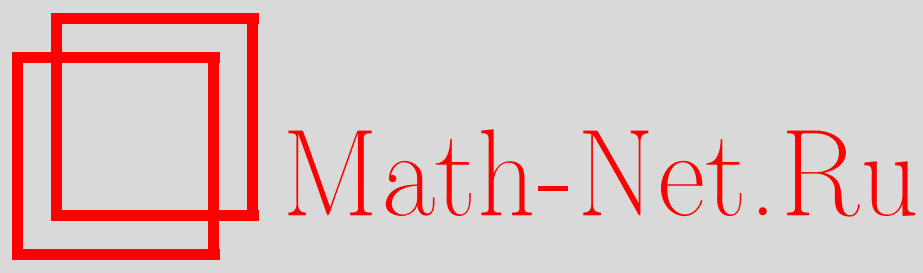

М. Л. Гандариас, М. С. Брузон, Симметрийный анализ и точные решения для некоторых уравнений Островского, ТМФ, 2011, том 168, номер 1, 49-64

DOI: https://doi.org/10.4213/tmf6663

Использование Общероссийского математического портала Math-Net.Ru подразумевает, что вы прочитали и согласны с пользовательским соглашением http://www . mathnet.ru/rus/agreement

Параметры загрузки:

IP: 44.207 .124 .84

26 апреля 2023 г., 03:31:46

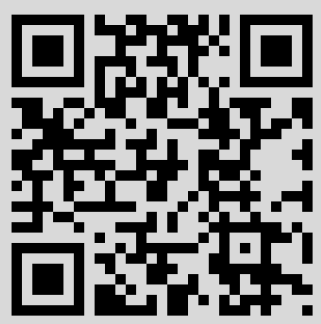




\section{СИММЕТРИЙНЫЙ АНАЛИЗ И ТОЧНЫЕ РЕШЕНИЯ ДЛЯ НЕКОТОРЫХ УРАВНЕНИЙ ОСТРОВСКОГО}

Обобщенное уравнение Островского и интегрируемое уравнение Вахненко, которое, как было доказано Вахненко и Паркером, эквивалентно редуцированному уравнению Островского, исследованы с помощью классического метода Ли, а также неклассического метода. С использованием простого нелинейного обыкновенного дифференциального уравнения найдено, что для некоторых многочленов по скорости обобщенное уравнение Островского обладает изобилием точных решений, которые можно выразить в терминах эллиптических функций Якоби, и, следовательно, обладает изобилием решений в виде периодических волн, уединенных волн, компактонов и т.д. Неклассический метод, примененный к соответствующей потенциальной системе для уравнения Вахненко, дает решения, которые не возникают ни из неклассических симметрий уравнения Вахненко, ни из потенциальных симметрий. Некоторые из этих уравнений демонстрируют интересное поведение, такое как "нелинейная суперпозиция".

Ключевые слова: классические симметрии, точные решения, уравнения в частных производных.

\section{1. ВВЕДЕНИЕ}

Для описания длинных внутренних волн во вращающемся океане Островский [1] вывел приближенное нелинейное уравнение

$$
\left(u_{t}+\left(u^{2}\right)_{x}-\beta u_{x x x}\right)_{x}=\gamma u,
$$

где $x \in \mathbb{R}$, а $\beta$ и $\gamma>0$ - коэффициенты дисперсии. Исследование уравнения Островского (УО) проводилось во многих работах (см. обзоры [2], [3] и библиографию в них). Были построены некоторые приближенные стационарные решения [4] (точных решений неизвестно), и исследована динамика отдельных нестационарных возмущений солитонного типа [5]. Уравнения типа УО представляют собой модель слабонелинейных длинных внутренних и поверхностных волн во вращающемся океане. В работах [6], [7] было показано, что уравнение (1) не имеет решений в виде

*Departamento de Matematicas, Universidad de Cadiz, Cadiz, Spain.

E-mail: marialuz.gandariaS@uca.es 
стационарных уединенных волн. Для длинных волн можно пренебречь высокочастотной дисперсией, $\beta=0$, и уравнение (1) принимает вид так называемого редуцированного уравнения Островского (РУО). Это уравнение рассматривалось многими авторами (см. работу [8] и библиографию в ней). В работе [9] доказано, что РУО можно преобразовать к новому интегрируемому уравнению

$$
u u_{x x t}-u_{x} u_{x t}+u^{2} u_{t}=0
$$

которое известно как уравнение Вахненко (УВ). В работе [10] использовались tanhи sine-cosine-методы для построения точных периодических и солитонных решений нелинейных эволюционных уравнений вида (2).

Вследствие сложности описания однонаправленного распространения во вращающейся жидкости скорости изменения дисперсионного и конвективного членов обычно пропорциональны многочлену по скорости. В работе [11] введено обобщенное уравнение Островского (ОУО) с многочленом по скорости

$$
\left(u_{t}+\left(u^{2}\right)_{x}-\beta u_{x x x}\right)_{x}=g(u),
$$

где $g(u)$ - многочлен по скорости, а именно $g(u)=\sum_{i=1}^{n} u^{i}$, а $n$ - константа. При $g(u)=u$ уравнение (3) представляет собой УО. В работе [11] показано, что в случае, когда указанный многочлен является квадратичным по скорости, т. е. $g(u)=u^{2}+u$, ОУО имеет новые решения в виде уединенных волн и преобразования Беклунда. Авторы работы [11] показали, что ОУО имеет большое количество точных решений в виде уединенных волн, таких как компактонное решение, многокомпактонное решение и компактоподобное кинковое решение. Используя метод баланса однородности, они вывели преобразование Беклунда типа ОУО и некоторые новые уединенные решения, в частности двойной симметричный пикон и двойные сингулярные уединенные решения.

Было известно несколько обобщений классического метода групп Ли для симметрийных редукций. Блуман и Коул [12] развили неклассический метод исследования симметрийных редукций уравнения теплопроводности, в работе [13] представлен алгоритм вычисления определяющих уравнений в рамках неклассического метода. Основная идея метода заключается в том, что дифференциальное уравнение в частных производных (ДУЧП) дополняется условием инвариантной поверхности

$$
\Phi \equiv \xi u_{x}+\tau u_{t}-\phi=0
$$

которое связано с векторным полем

$$
\mathbf{v}=\xi \partial_{x}+\tau \partial_{t}+\phi \partial_{u}
$$

Если потребовать, чтобы как уравнение (3) (или (2)), так и уравнение (4) были инвариантны относительно преобразования с инфинитезимальным генератором (5), то получится переопределенная нелинейная система уравнений для инфинитезимальных элементов $\xi(x, t, u), \tau(x, t, u)$ и $\phi(x, t, u)$. Число определяющих уравнений, возникающих в неклассическом методе, меньше, чем в классическом методе, а следовательно, множество решений в общем случае больше, чем в классическом методе, поскольку в данном методе требуется только, чтобы подмножество решений 
уравнений (3) (или (2)) и (4) было инвариантно относительно инфинитезимального генератора (5).

В настоящей работе исследуются уравнения (2) и (3) с точки зрения теории симметрийных редукций ДУЧП. Получены классические симметрии, допускаемые уравнениями (2) и (3) для произвольных $g$. Получен вид функции $g$, для которого уравнение (3) допускает дополнительные классические симметрии; для редукции уравнений к обыкновенным дифференциальным уравнениям (ОДУ) затем используются групповые преобразования. В настоящей работе также рассмотрены неклассические симметрии уравнений (3) и (2) с использованием метода из работы [12]. Все определяющие уравнения были получены с помощью пакета МАХIMA symmgrp2009.max, недавно модифицированного Хереманном.

В работе [14] был предложен простейший метод поиска точных решений нелинейных дифференциальных уравнений. Там было указано, что многие из так называемых "новых решений в виде бегущей волны" можно вывести из решений простого нелинейного ОДУ. В настоящей работе в процессе поиска решений в виде бегущих волн было показано, что в случае, когда многочлен по скорости представляет собой квинтику, т. е. $f(u)=k_{5} u^{5}+k_{4} u^{4}+k_{3} u^{3}+k_{2} u^{2}+k_{1} u+k_{0}$, у ОУО имеется изобилие точных решений, которые можно выразить в терминах эллиптических функций Якоби, и, следовательно, ОУО имеет богатый набор точных решений, которые можно выразить в терминах тригонометрических и гиперболических функций. Поэтому у уравнения (3) имеется изобилие решений в виде периодических волн, уединенных волн, компактонов и т.д. Эти решения выводятся из решений простого нелинейного ОДУ [15].

Очевидным ограничением теоретико-групповых методов, основанных на локальных симметриях, является тот факт, что многие ДУЧП не имеют локальных симметрий. Оказывается, что ДУЧП могут допускать нелокальные симметрии, инфинитезимальные генераторы которых зависят от интегралов по зависимым переменным некоторым специальным образом. В работах [16]-[18] был приведен критерий, которому должны удовлетворять нелокальные симметрии ДУЧП, реализованные в виде локальных симметрий системы ДУЧП, которая "накрывает" заданное ДУЧП. В работе [19] приведены нетривиальные примеры нелокальных симметрий, следующих из эвристических процедур.

В работе [20] введен метод нахождения нового класса симметрий для ДУЧП. Предположим, что заданное скалярное ДУЧП второго порядка

$$
F\left(x, t, u, u_{x}, u_{t}, u_{x x}, u_{x t}, u_{t t}\right)=0 \text {, }
$$

где нижние индексы обозначают частные производные, можно записать в виде закона сохранения

$$
\frac{D}{D t} f\left(x, t, u, u_{x}, u_{t}\right)-\frac{D}{D x} g\left(x, t, u, u_{x}, u_{t}\right)=0
$$

для некоторых функций $f$ и $g$ указанных аргументов. Здесь $D / D x$ и $D / D t$ - операторы полных производных, которые определяются как

$$
\frac{D}{D x}=\frac{\partial}{\partial x}+u_{x} \frac{\partial}{\partial u}+u_{x x} \frac{\partial}{\partial u_{x}}+u_{x t} \frac{\partial}{\partial u_{t}}+\cdots,
$$




$$
\frac{D}{D t}=\frac{\partial}{\partial t}+u_{t} \frac{\partial}{\partial u}+u_{x t} \frac{\partial}{\partial u_{x}}+u_{t t} \frac{\partial}{\partial u_{t}}+\cdots
$$

Используя закон сохранения (6), можно ввести вспомогательную потенциальную переменную $v$ и составить вспомогательную потенциальную систему

$$
v_{x}=f\left(x, t, u, u_{x}, u_{t}\right), \quad v_{t}=g\left(x, t, u, u_{x}, u_{t}\right) .
$$

Для многих уравнений, используемых в физике, можно исключить переменную $u$ из потенциальной симметрии (7) и получить вспомогательное интегрируемое или потенциальное уравнение (метод интегрируемых уравнений)

$$
G\left(x, t, v, v_{x}, v_{t}, v_{x x}, v_{x t}, v_{t t}\right)=0
$$

для некоторой функции $G$ с указанными аргументами. Любая группа Ли точечных преобразований

$$
\mathbf{w}=\xi(x, t, u, v) \partial_{x}+\tau(x, t, u, v) \partial_{t}+\phi(x, t, u, v) \partial_{u}+\psi(x, t, u, v) \partial_{v},
$$

допускаемая системой (7), приводит к потенциальной симметрии заданного ДУЧП (6) тогда и только тогда, когда выполнено условие

$$
\xi_{v}^{2}+\tau_{v}^{2}+\phi_{v}^{2} \neq 0
$$

В работе [21] неклассический метод был расширен до потенциальной системы (7) (алгоритм I), а также до интегрируемого уравнения (алгоритм II).

Алгоритм I. Неклассический метод применяется к соответствующей потенциальной системе (7). Любая группа Ли точечных преобразований (9), которую допускает система (7), приводит к потенииальной симметрии заданного ДУЧП (6), если выполнено условие (10).

АЛГОРИТМ II: НЕКЛАССИЧЕСКИЙ МЕТОД ИССЛЕДОВАНИЯ ПОТЕНЦИАЛЬНЫХ уРАВНЕНИй. Неклассический метод применяется к соответствующему потенциальному уравнению (8). Любая группа Ли точечных преобразований

$$
X=\xi(x, t v) \frac{\partial}{\partial x}+\tau(x, t, v) \frac{\partial}{\partial t}+\psi(x, t, v) \frac{\partial}{\partial v},
$$

которая допускает уравнение (8), приводит к потенииальной симметрии заданного ДУЧП (6), если выполнено условие

$$
\xi_{v}^{2}+\tau_{v}^{2} \neq 0
$$

В работе [21] было указано, что неклассический метод, будучи применен к потенциальной системе (алгоритм I), часто дает набор неопределенных определяющих уравнений, в то время как этот метод гораздо проще применить к потенциальному уравнению (алгоритм II). Однако в работе [22] было доказано, что существенным недостатком алгоритма II является тот факт, что соответствующее условие (11) есть достаточное, но не необходимое условие для того, чтобы определить, является ли генератор неклассическим потенциальным генератором. В работе [23] был 
предложен модифицированный алгоритм I. C использованием этого алгоритма в работе [22] были получены некоторые неклассические потенциальные симметрии для уравнения Бюргерса.

В настоящей работе рассматриваются симметрийные редукции и точные решения РУО. Классический и неклассический методы применяются к РУО и УВ, а также к естественной связанной с ними потенциальной системе. Доказано, что неклассический метод (при $\tau=1)$, примененный к уравнению (2), дает редукции, эквивалентные редукциям, выведенным с использованием классического метода Ли. Тем не менее неклассический метод, будучи применен к потенциальной системе, соответствующей (2), дает новые генераторы. Неклассические симметрийные редукции, полученные для системы

$$
v_{x}=u, \quad v_{t}=-\frac{u_{t x}}{u},
$$

порождают изобилие аналитических решений уравнения (2). Некоторые из этих решений демонстрируют интересное поведение, такое как "нелинейная суперпозиция".

Заметим, что хотя инфинитезимальные элементы не зависят от $v=\int u(x) d x$ и не соответствуют неклассическим потенциальным генераторам, они не проецируются ни на какой из инфинитезимальных элементов, соответствующих классическим или неклассическим генераторам уравнения (2); следовательно, и является новым точным решением уравнения (2), которое нельзя получить с использованием классических или неклассических симметрий уравнения (2).

\section{2. КЛАССИЧЕСКИЕ СИММЕТРИИ ДЛЯ ОУО}

В данном разделе выполняется симметрийный анализ Ли для ОУО (3). Рассмотрим однопараметрическую группу Ли инфинитезимальных преобразований по $(x, t, u)$, задаваемых в виде

$$
\begin{aligned}
x^{*} & =x+\varepsilon \xi(x, t, u)+O\left(\varepsilon^{2}\right), \\
t^{*} & =t+\varepsilon \tau(x, t, u)+O\left(\varepsilon^{2}\right), \\
u^{*} & =u+\varepsilon \phi(x, t, u)+O\left(\varepsilon^{2}\right),
\end{aligned}
$$

где $\varepsilon$ - групповой параметр. Далее потребуем, чтобы это преобразование оставляло инвариантным множество решений уравнения (3). Это приводит к переопределенной линейной системе из одиннадцати уравнений для инфинитезимальных элементов $\xi(x, t, u), \tau(x, t, u)$ и $\phi(x, t, u)$. Соответствующая алгебра Ли инфинитезимальных симметрий представляет собой множество векторных полей вида (5). Если определены инфинитезимальные элементы, то симметрийные переменные находятся путем решения условия инвариантной поверхности (4).

Решая эту систему, находим, что если $g$ - произвольная функция, то единственные симметрии, допускаемые уравнением (3), это

$$
\mathbf{v}_{1}=\frac{\partial}{\partial x}, \quad \mathbf{v}_{2}=\frac{\partial}{\partial t} .
$$

Единственный функциональный вид для $g$ при условии $g \neq$ const, обладающий дополнительными симметриями, есть

$$
g(u)=(a u+c)^{3} .
$$


Для этого специального вида $g$ получаем, что допускаемая группа симметрий имеет генераторы

$$
\mathbf{v}_{1}, \quad \mathbf{v}_{2}, \quad \mathbf{v}_{3}=\left(x-\frac{4 c}{a}\right) \frac{\partial}{\partial x}+3 t \frac{\partial}{\partial t}-2\left(u+\frac{2 c}{a}\right) \frac{\partial}{\partial u} .
$$

Теперь рассмотрим следующие редукции, которые возникают из векторных полей $\lambda \mathbf{v}_{1}+\mathbf{v}_{2}$ и $\mathbf{v}_{3}$.

РЕдукция 1. Используя генератор $\mathbf{v}_{1}+\lambda \mathbf{v}_{2}$, получаем переменные подобия и решение подобия:

$$
z=x-\lambda t, \quad u=h(z)
$$

и ОДУ

$$
h^{\prime \prime \prime \prime}+2 h h^{\prime \prime}-\lambda h^{\prime \prime}+2\left(h^{\prime}\right)^{2}=g(h) .
$$

РЕдукция 2. Используя генератор $\mathbf{v}_{3}$, получаем переменные подобия и решение подобия:

$$
z=-\frac{x}{t^{1 / 3}}+\frac{2 c t^{2 / 3}}{a}, \quad u=\frac{h}{t^{2 / 3}}-\frac{c}{a}
$$

и ОДУ

$$
3 b h^{\prime \prime \prime \prime}+(z-6 h) h^{\prime \prime}-6\left(h^{\prime}\right)^{2}+3 h^{\prime}+3 a^{3} \gamma h^{3}=0
$$

\section{3. НЕКЛАССИЧЕСКИЕ СИММЕТРИИ ДЛЯ ОУО}

Потребовав, чтобы как уравнение (3), так и уравнение (4) были инвариантны относительно преобразования с инфинитезимальным генератором (5), получаем переопределенную нелинейную систему уравнений для инфинитезимальных элементов $\xi(x, t, u), \tau(x, t, u)$ и $\phi(x, t, u)$. Можно выделить два случая.

В случае $\tau \neq 0$ без потери общности можно положить $\tau=1$. Применение неклассического метода к уравнению (3) приводит к появлению следующих семи определяющих уравнений для инфинитезимальных элементов:

$$
\begin{gathered}
\xi_{u}=0, \\
\phi_{u u}=0, \\
2 \phi_{u x}-3 \xi_{x x}=0, \\
2 \xi_{x}+\phi_{u}=0, \\
-4 b \xi_{x x x}+3 \xi \xi_{x}-4 u \xi_{x}+\xi_{t}+6 b \phi_{u x x}-2 \phi=0, \\
b \xi_{x x x x}+\xi \xi_{x x}-2 u \xi_{x x}-2 \phi_{u x} \xi+4 \phi_{u x} u+4 \phi_{x}-4 b \phi_{u x x x}=0, \\
-4 \phi_{x} \xi_{x}+4 g \xi_{x}-2 \phi_{x x} u+b \phi_{x x x x}+\phi_{u} \phi_{x}-\phi \phi_{u x}-g \phi_{u}-\phi_{t x}+g_{u} \phi=0 .
\end{gathered}
$$

Решая эту систему, получаем, что $\xi=f_{1} x+f_{2}, \phi=\beta-2 u d \xi / d x$, где $f_{i}(t), i=1,2$, $\beta(t)$ и $g(u)$ должны удовлетворять уравнениям

$$
\begin{gathered}
-2 \frac{d g}{d u} f_{1} u+6 g f_{1}+\beta \frac{d g}{d u}=0, \\
\frac{d f_{1}}{d t} x+3 f_{1}^{2} x+\frac{d f_{2}}{d t}+3 f_{1} f_{2}-2 \beta=0 .
\end{gathered}
$$

Решая эти уравнения, получаем

$$
\beta=-\frac{\sqrt[3]{k_{4}}}{6 t}, \quad f_{2}=\frac{k_{5}}{t}-\frac{\sqrt[3]{k_{4}}}{3}, \quad g(u)=k_{6}\left(64 u^{3}+48 \sqrt[3]{k_{4}} u^{2}+12 k_{4}^{2 / 3} u+k_{4}\right) .
$$


Следовательно, после решения определяющей системы можно убедиться, что при $\tau \neq 0$ мы лишь воспроизводим классические симметрии и что произведена полная классификация неклассических симметрий ОУО. Можно утверждать, что уравнение (3) не допускает собственных неклассических симметрий при $\tau=1$ для любой функции $g(u)$.

В случае $\tau=0$ без потери общности можно положить $\xi=1$; тогда получим только условие для ф с 45 слагаемыми.

Таким образом, чтобы двигаться вперед, мы предложим несколько подстановок для функции $\phi(x, t, u)$.

1. Для произвольной функции $g(u)$ получаем произвольную функцию $\phi=\phi(x, t)$.

2. При $g(u)=-b k_{1}^{4} n(2 n-1)(3 n-2) u^{4 n-3}+k_{2} u^{2 n-1}+2 k_{1}^{2}(n+1) u^{2 n}$ получаем $\phi=k_{1} u^{n}$.

3. При $g(u)=4 k_{1}^{2} u^{2}+k_{2} u$ получаем $\phi=k_{1} u$. $\mathrm{K}$ сожалению, эти генераторы приводят к разделению переменных, и новых редукций не получается.

\section{4. РЕШЕНИЯ В ВИДЕ БЕГУЩИХ ВОЛН}

Авторы работы [11] получили, что ОУО с многочленом по скорости вида $g(u)=$ $u^{2}+u$ обладает изобилием точных решений в виде уединенных волн, таких как компактонное решение, многокомпактонное решение и компактоподобное кинковое решение.

Поскольку уравнение (3) допускает дополнительные симметрии тогда и только тогда, когда $g(u)$ является кубическим многочленом $f(u)=(a u+c)^{3}$, а предыдущие результаты получены в работе [11] для функции $g$, являющейся квадратичным многочленом, будем искать решения ОУО в виде бегущей волны в случае, когда $g(u)-$ многочлен вида $g(u)=\sum_{i=1}^{n} k_{i} u^{i}$. Тогда редуцированное ОДУ принимает вид

$$
-\beta h^{\prime \prime \prime \prime}+2 h h^{\prime \prime}-\lambda h^{\prime \prime}+2\left(h^{\prime}\right)^{2}=g(h),
$$

где $g(h)=\sum_{i=1}^{n} k_{i} h^{i}$. Полагая $n=2 / \beta$, получаем следующую теорему.

Теорема. Уравнение $(21)$, в котором

$$
g(h)=a_{5} h^{\frac{4}{\beta}+1}+a_{7} h^{\frac{2}{\beta}+2}+a_{3} h^{\frac{2}{\beta}+1}+\frac{a_{8}}{h^{\frac{2}{\beta}-2}}+\frac{a_{4}}{h^{\frac{2}{\beta}-1}}+\frac{a_{6}}{h^{\frac{4}{\beta}-1}}+a_{2} h^{2}+a_{1} h,
$$

допускает любые решения в терминах эллиптических функиий Якоби:

1) $h=\alpha \operatorname{sn}^{\beta}(k z, p)$, где $a_{i}, i=0, \ldots, 8$, имеют вид

$$
\begin{aligned}
a_{1}= & \beta^{2} k^{2} \mu\left(p \lambda+\lambda-b \beta^{2} k^{2} \mu^{3} p^{2}-4 b \beta^{2} k^{2} \mu^{3} p-10 b k^{2} \mu^{3} p-b \beta^{2} k^{2} \mu^{3}\right), \\
a_{2}= & -4 \beta^{2} k^{2} \mu^{2}(p+1), \\
a_{3}= & -\frac{\beta(\beta+1) k^{2} \mu p\left(\lambda-2 b \beta^{2} k^{2} \mu^{3} p-4 b \beta k^{2} \mu^{3} p-4 b k^{2} \mu^{3} p-2 b \beta^{2} k^{2} \mu^{3}-4 b \beta k^{2} \mu^{3}-4 b k^{2} \mu^{3}\right)}{\alpha^{2 / \beta}}, \\
a_{4}= & -\alpha^{2 / \beta}(\beta-1) \beta k^{2} \mu \times \\
& \times\left(\lambda-2 b \beta^{2} k^{2} \mu^{3} p+4 b \beta k^{2} \mu^{3} p-4 b k^{2} \mu^{3} p-2 b \beta^{2} k^{2} \mu^{3}+4 b \beta k^{2} \mu^{3}-4 b k^{2} \mu^{3}\right), \quad(22) \\
a_{5}= & -\frac{b \beta(\beta+1)(\beta+2)(\beta+3) k^{4} \mu^{4} p^{2}}{\alpha^{4 / \beta}}, \\
a_{6}= & -\alpha^{4 / \beta} b(\beta-3)(\beta-2)(\beta-1) \beta k^{4} \mu^{4},
\end{aligned}
$$




$$
\begin{aligned}
& a_{7}= \frac{2 \beta(2 \beta+1) k^{2} \mu^{2} p}{\alpha^{2 / \beta},} \\
& a_{8}= 2 \alpha^{2 / \beta} \beta(2 \beta-1) k^{2} \mu^{2} ; \\
&2) h=\alpha \mathrm{cn}^{\beta}(k z, p), \text { где } a_{i}, i=0, \ldots, 8, \text { имеют вид } \\
& a_{1}=-\beta^{2} k^{2} \mu\left(2 p \lambda-\lambda+6 b \beta^{2} k^{2} \mu^{3} p^{2}+10 b k^{2} \mu^{3} p^{2}-6 b \beta^{2} k^{2} \mu^{3} p-10 b k^{2} \mu^{3} p+b \beta^{2} k^{2} \mu^{3}\right), \\
& a_{2}= 4 \beta^{2} k^{2} \mu^{2}(2 p-1), \\
& a_{3}= \frac{\beta(\beta+1) k^{2} \mu p\left(\lambda+4 b \beta^{2} k^{2} \mu^{3} p+8 b \beta k^{2} \mu^{3} p+8 b k^{2} \mu^{3} p-2 b \beta^{2} k^{2} \mu^{3}-4 b \beta k^{2} \mu^{3}-4 b k^{2} \mu^{3}\right),}{\alpha^{2 / \beta}} \\
& a_{4}= \alpha^{2 / \beta}(\beta-1) \beta k^{2} \mu(p-1) \times \\
& \times\left(\lambda+4 b \beta^{2} k^{2} \mu^{3} p-8 b \beta k^{2} \mu^{3} p+8 b k^{2} \mu^{3} p-2 b \beta^{2} k^{2} \mu^{3}+4 b \beta k^{2} \mu^{3}-4 b k^{2} \mu^{3}\right), \quad(23) \\
& a_{5}=-\frac{b \beta(\beta+1)(\beta+2)(\beta+3) k^{4} \mu^{4} p^{2}}{\alpha^{4 / \beta}}, \\
& a_{6}=-\alpha^{4 / \beta} b(\beta-3)(\beta-2)(\beta-1) \beta k^{4} \mu^{4}(p-1)^{2}, \\
& a_{7}=-\frac{2 \beta(2 \beta+1) k^{2} \mu^{2} p}{\alpha^{2 / \beta}}, \\
& a_{8}=-2 \alpha^{2 / \beta} \beta(2 \beta-1) k^{2} \mu^{2}(p-1) .
\end{aligned}
$$

ДокАЗАтЕльство. Потребуем, чтобы уравнение (21) удовлетворялось тождественно, подставим соответствующее выражение $h=\alpha \operatorname{sn}^{\beta}(k z, p)$ или $h=\alpha \mathrm{cn}^{\beta}(k z, p)$ и его производные в (21), тогда, собирая коэффициенты при различных степенях $h$ и приравнивая их к нулю, получаем $a_{i}, i=0, \ldots, 8$.

В качестве частных случаев получаем, что при

$$
g(u)=-4 h k^{2} \mu\left(\lambda+4 b k^{2} \mu^{3}\right)-\frac{h^{2}\left(\lambda+4 b k^{2} \mu^{3}\right)}{b \mu}
$$

имеем

$$
h=-6 b k^{2} \mu^{2} \operatorname{sech}^{2}(k z),
$$

что представляет собой гладкое солитонное решение, а при

$$
g(u)=16 h^{2} k^{2} \mu^{2}-\frac{2 k^{2}\left(\lambda+4 b k^{2} \mu^{3}\right)^{2}}{3}
$$

имеем

$$
h=-\frac{\operatorname{ch}^{2}(k z)\left(\lambda+4 b k^{2} \mu^{3}\right)}{3 \mu} .
$$

\section{5. РЕШЕНИЯ В ВИДЕ БЕГУЩИХ И УЕДИНЕННЫХ ВОЛН}

Любое решение $h$ уравнения (16) будет определять решение исходного уравнения (3) в виде бегущей волны. Полагая

$$
g(u)=k_{3} u^{3}+k_{2} u^{2}+k_{1} u+k_{0},
$$

получаем солитонное решение (см. рис. 1)

$$
u=k \operatorname{sech}^{2}(x-\lambda t),
$$




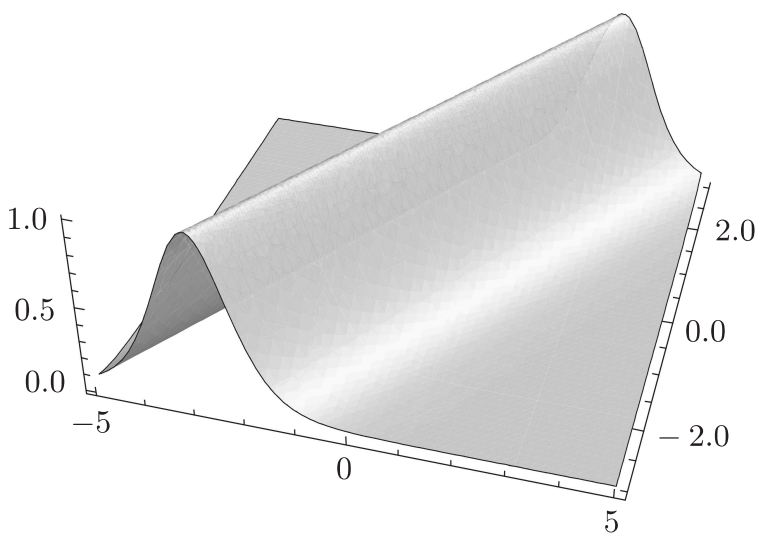

Рис. 1. Солитонное решение (24).

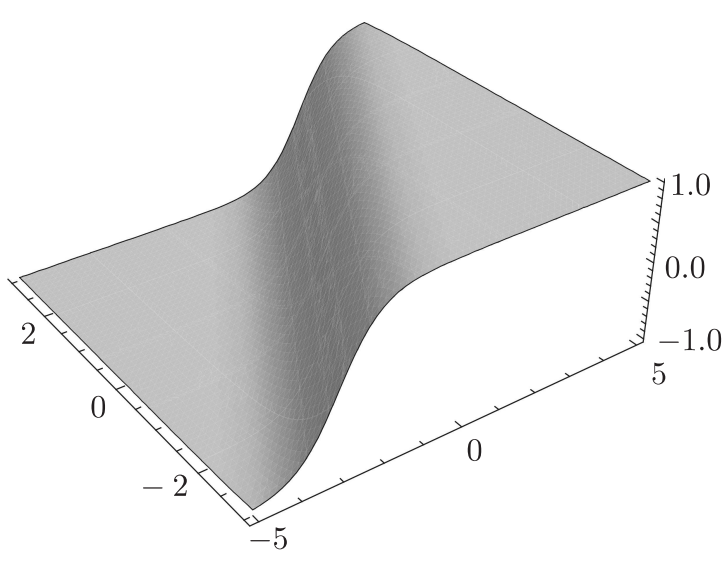

Рис. 2. Кинковое решение (25).

где

$$
k_{0}=0, \quad k_{1}=-4 \lambda-16 \beta, \quad k_{2}=\frac{6 \lambda}{k}+\frac{120 \beta}{k}+16, \quad k_{3}=-\frac{20}{k}-\frac{120 \beta}{k^{2}} .
$$

Полагая

$$
g(u)=k_{5} u^{5}+k_{4} u^{4}+k_{3} u^{3}+k_{2} u^{2}+k_{1} u+k_{0},
$$

получаем кинковое решение (см. рис. 2)

$$
u=k \operatorname{th}(x-\lambda t)
$$

где

$k_{0}=2 k^{2}, \quad k_{1}=2 \lambda-16 \beta, \quad k_{2}=-8, \quad k_{3}=\frac{40 \beta-2 \lambda}{k^{2}}, \quad k_{4}=\frac{6}{k^{2}}, \quad k_{5}=-\frac{24 \beta}{k^{4}}$.

Полагая

$$
\begin{aligned}
g(u)= & 360 \sqrt[3]{u} k_{1}^{4} k_{2}^{2 / 3}-30 u^{2 / 3} k_{1}^{2}\left(52 k_{1}^{2}+\lambda\right) k_{2}^{1 / 3}+132 u^{5 / 3} k_{1}^{2} k_{2}^{1 / 3}+ \\
& +36 u k_{1}^{2}\left(36 k_{1}^{2}+\lambda\right)-144 u^{2} k_{1}^{2}
\end{aligned}
$$




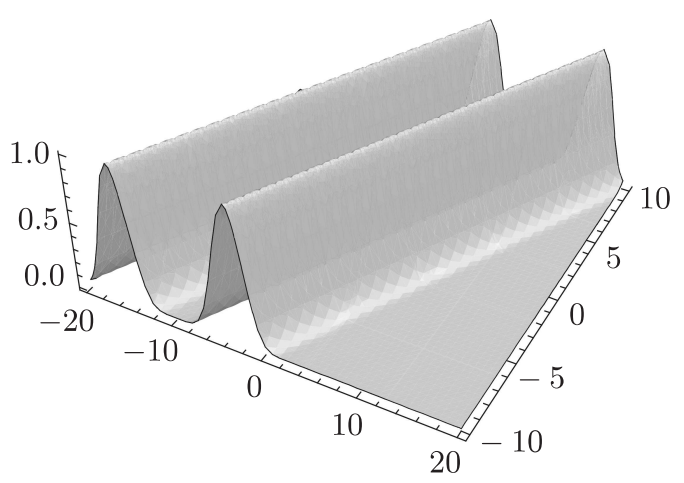

Рис. 3. Компактонное решение (26).

получаем компактонное решение

$$
u=k_{2} \cos ^{6}\left(k_{1}(x-\lambda t)\right)
$$

а при

$$
g=\frac{360 h^{1 / 3} k^{2 / 3}}{2^{2 / 3}}+\frac{132 h^{5 / 3} k^{1 / 3}}{2^{1 / 3}}-\frac{30(c+52) h^{2 / 3} k^{1 / 3}}{2^{1 / 3}}-144 h^{2}+36(c+36) h
$$

получаем компактонное решение (см. рис. 3)

$$
u=\frac{k \sin ^{6}(x-t)}{2} .
$$

\section{6. КЛАССИЧЕСКИЕ СИММЕТРИИ ДЛЯ УВ}

Применение классического метода Ли к уравнению (2) приводит к переопределенной линейной системе из 11 уравнений для инфинитезимальных элементов $\xi(x, t, u)$, $\tau(x, t, u)$ и $\phi(x, t, u)$. Решая эту систему, можно легко получить следующие инфинитезимальные элементы:

$$
\xi=k_{1} x+k_{2}, \quad \tau=\alpha(t), \quad \phi=-2 k_{1} u,
$$

где $\alpha(t)$ - произвольная функция, а $k_{1}$ и $k_{2}$ - произвольные константы. Соответствующие векторные поля имеют вид

$$
\mathbf{v}_{1}=\frac{\partial}{\partial x}, \quad \mathbf{v}_{2}=\alpha(t) \frac{\partial}{\partial t}, \quad \mathbf{v}_{3}=x \frac{\partial}{\partial x}-2 u \frac{\partial}{\partial u} .
$$

Решая уравнение (4), получаем две канонические симметрийные редукции.

РЕдУкция 1. $k_{1} \neq 0$. Используя генератор $k_{1} \mathbf{v}_{3}+k_{2} \mathbf{v}_{1}+\mathbf{v}_{2}$, можно положить $\alpha(t)=\beta(t) / \beta^{\prime}(t), k_{1}=1$ и $k_{2}=0$ (где $\left.f^{\prime} \equiv d f / d t\right)$. Таким образом, получаем симметрийную редукцию

$$
z=\frac{x}{\beta(t)}, \quad u=\frac{h(z)}{\beta^{2}(t)},
$$


где для $h(z)$ выполнено уравнение

$$
-z h h^{\prime \prime \prime}+z h^{\prime} h^{\prime \prime}-z h^{2} h^{\prime}-4 h h^{\prime \prime}+3\left(h^{\prime}\right)^{2}-2 h^{3}=0 .
$$

РЕдукция $2 . k_{1}=0$. Используя генератор $\mathbf{v}_{1}+\mathbf{v}_{2}$, получаем переменные подобия и решения подобия:

$$
z=x-\beta(t), \quad u=h(z),
$$

где для $h(z)$ выполнено уравнение

$$
h h^{\prime \prime \prime}-h^{\prime} h^{\prime \prime}+h^{2} h^{\prime}=0 .
$$

Заметим, что, поделив на $h^{2}$ и один раз проинтегрировав по $z$, можно привести уравнение (31) к виду

$$
h^{\prime \prime}+h^{2}=k h \text {. }
$$

Уравнение (32) допускает решения в терминах эллиптических функций Якоби. Таким образом, получаем следующие решения уравнения (31).

Случай 1. Решение

$$
h=a_{2} \operatorname{sn}^{2}(k z, p)+\frac{b_{2}}{\operatorname{sn}^{2}(k z, p)}+a_{0},
$$

где

$$
a_{2}=b_{2}=-6 k^{2} p, \quad a_{0}=2 k^{2}\left(\sqrt{p^{2}+14 p+1}+p+1\right) .
$$

При специальных значениях $p$ получаем

$$
\begin{array}{ll}
h=-\frac{6 k^{2}}{\operatorname{ch}^{2}(k z) \operatorname{sh}^{2}(k z)}, & p=1, \\
h=4 k^{2}-\frac{6 k^{2}}{\sin ^{2}(k z)}, & p=0 .
\end{array}
$$

Случай 2. Решение

$$
h=\frac{b_{2}}{\operatorname{sn}^{2}(k z, p)}+a_{0}
$$

где

$$
\begin{gathered}
b_{2}=-6 k^{2}, \quad a_{0}=-\frac{2 k^{2} p-6 k^{4}-4 k^{2}+d}{2}, \\
d^{2}=-4 k^{2}\left(5 k^{2} p^{2}-18 k^{4} p+4 k^{2} p+3 d p+9 k^{6}-3 d k^{2}-4 k^{2}\right) .
\end{gathered}
$$

Случай 3. Решение

$$
h=a_{2} \operatorname{sn}^{2}(k z, p)+a_{0},
$$

где

$$
a_{2}=-6 k^{2} p, \quad a_{0}=-2 k^{2} \sqrt{p^{2}-p+1}+2 k^{2}(p+1) .
$$



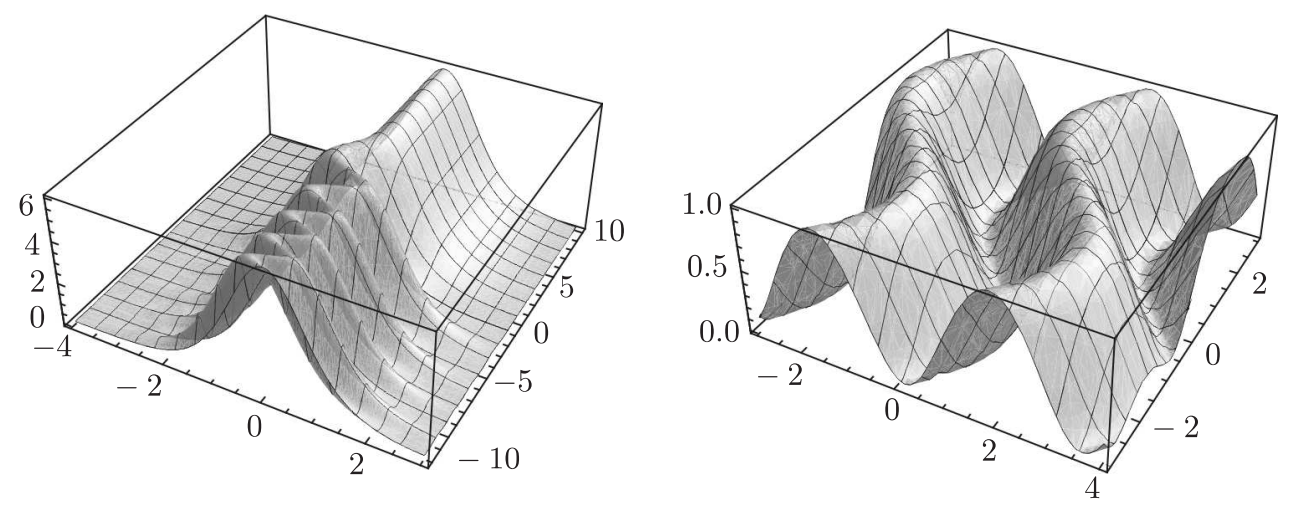

Рис. 4. Решения (40).

При специальных значениях $p$ получаем

$$
\begin{array}{ll}
h=6 k^{2} \operatorname{sech}^{2}(k z), & p=1, \\
h=4 k^{2}-\frac{6 k^{2}}{\sin ^{2}(k z)}, & p=0 .
\end{array}
$$

Из решений уравнения (31), используя (30), получаем следующие решения уравнения (2) в терминах эллиптических функций Якоби:

$$
\begin{aligned}
& u=a_{2} \operatorname{sn}^{2}(k(x-\beta(t)), p)+\frac{b_{2}}{\operatorname{sn}^{2}(k(x-\beta(t)), p)}+a_{0}, \\
& u=\frac{b_{2}}{\operatorname{sn}^{2}(k(x-\beta(t)), p)}+a_{0} \\
& u=a_{2} \operatorname{sn}^{2}(k(x-\beta(t)), p)+a_{0}
\end{aligned}
$$

и соответствующие решения в терминах вырожденных тригонометрических и гиперболических функций:

$$
\begin{aligned}
& u=-6 k^{2} \operatorname{sech}^{2}\left(k(x-\beta(t)) \operatorname{csch}^{2}(k(x-\beta(t))\right. \\
& u=4 k^{2}-6 k^{2} \operatorname{csch}^{2}(k(x-\beta(t)) \\
& u=6 k^{2} \operatorname{sech}^{2}(k(x-\beta(t))
\end{aligned}
$$

Некоторые частные случаи этих решений при $\beta(t)=\lambda t$ были получены в работе [10] с использованием tanh-метода, а также sine-cosine-метода (см. рис. 4):

$$
u=6 \operatorname{sech}^{2}(x-\operatorname{Ai}(t)), \quad u=\operatorname{sn}(x+\sin t, 1 / 3)^{2} .
$$

\section{7. НЕКЛАССИЧЕСКИЕ СИММЕТРИИ ДЛЯ УВ}

Для получения неклассических симметрий уравнения (2) применим алгоритм решения определяющих уравнений, описанный в работе [23]. Можно выделить два случая. 
В случае $\tau \neq 0$ без потери общности можно положить $\tau=1$. Применение неклассического метода к уравнению (2) приводит к появлению 20 определяющих уравнений для инфинитезимальных элементов. Из этих уравнений немедленно получаем

$$
\xi=\xi(x, t), \quad \phi=\alpha(x, t) u+\beta(x, t,) u^{1 / 3} .
$$

Здесь можно выделить ряд случаев.

Случай 1. Если $\xi=0$, получаем

$$
\phi=\alpha(x, t) u+\beta(x, t) u^{1 / 3},
$$

где $\alpha(x, t)$ и $\beta(x, t)$ - произвольные функции. Эти генераторы являются слишком общими для практического использования.

Случай 2. Если $\xi \neq 0$, то $\beta=0, \alpha=-2 \xi_{x}$, где $\xi$ должно удовлетворять уравнениям

$$
\begin{gathered}
2 \xi^{2} \xi_{x x}+3 \xi_{t} \xi_{x}-3 \xi \xi_{t x}=0, \\
-3 \xi^{2} \xi_{x x}-4 \xi_{t} \xi_{x}+4 \xi \xi_{t x}=0, \\
\xi_{t} \xi_{x}-\xi \xi_{t x}=0, \\
2 \xi \xi_{x} \xi_{x x x}+\xi_{t} \xi_{x x x}+3 \xi\left(\xi_{x x}\right)^{2}-\xi \xi_{t x x x}=0, \\
2 \xi^{2} \xi_{x x x}+5 \xi \xi_{x} \xi_{x x}+3 \xi_{t} \xi_{x x}-3 \xi \xi_{t x x}=0 .
\end{gathered}
$$

Из первых двух уравнений получаем $\xi=f_{1}(t) x+f_{2}(t)$. Здесь также можно выделить следующие случаи:

а) $\xi_{x} \neq 0, f_{1} \neq 0$, тогда

$$
\xi=f_{1}(t)\left(x+k_{1}\right), \quad \tau=1, \quad \phi=-2 f_{1}(t) u
$$

б) $\xi_{x}=0$, тогда

$$
\xi=f_{2}(t), \quad \tau=1, \quad \phi=0 .
$$

В обоих случаях инфинитезимальные элементы эквивалентны тем элементам, которые получаются с использованием классического метода.

\section{8. НЕКЛАССИЧЕСКИЕ ПОТЕНЦИАЛЬНЫЕ СИММЕТРИИ ДЛЯ УВ}

В работах [13], [24] предложен метод нахождения новых классов симметрий для ДУЧП, когда его можно записать в сохраняющемся виде.

Пусть уравнение (2) - это УВ. Чтобы найти потенциальные симметрии, запишем уравнение в сохраняющемся виде. Из этого сохраняющегося вида получается соответствующая вспомогательная система (12) с потенциалами в качестве дополнительных зависимых переменных. Если функции $u(x), v(x)$ удовлетворяют системе (12), то $u(x)$ есть решение уравнения (2), а $v(x)$ - решение уравнения

$$
v_{t} v_{x}+v_{x x t}=0 \text {. }
$$

Чтобы получить неклассические потенциальные симметрии для УВ, применим неклассический метод к уравнению (12). В случае $\tau \neq 0$ без потери общности можно 
положить $\tau(x, t, u, v)=1$. Применение неклассического метода к уравнению (12) приводит к появлению следующих определяющих уравнений:

$$
\begin{gathered}
\xi \xi_{u u}-\left(\xi_{u}\right)^{2}=0, \quad \psi_{u}-u \xi_{u}=0 \\
-\xi \xi_{u} \xi_{x}+\psi \xi_{u} \xi_{v}+\xi^{2} \xi_{v}-\phi \xi \xi_{u u}+\xi^{2} \xi_{u x}+2 u \xi^{2} \xi_{u v}- \\
-\psi \xi \xi_{u v}+\phi\left(\xi_{u}\right)^{2}+\xi_{t} \xi_{u}+\phi_{u} \xi \xi_{u}-\xi \xi_{t u}-\phi_{u u} \xi^{2}=0 \\
-u \xi_{x}-u^{2} \xi_{v}+\psi_{v} u+\psi_{x}-\phi=0 \\
u \xi \xi_{v} \xi_{x}-\psi \xi_{v} \xi_{x}-\phi \xi_{u} \xi_{x}-\xi_{t} \xi_{x}-u^{2} \xi^{2} \xi_{v v}-2 u \xi^{2} \xi_{v x}+ \\
+\psi \xi \xi_{v x}-\phi_{u} u \xi \xi_{v}+\phi_{u} \psi \xi_{v}+\phi \xi \xi_{u x}+2 u^{2} \xi^{2} \xi_{u}- \\
-3 \psi u \xi \xi_{u}+2 \phi_{v} u \xi \xi_{u}-\phi_{x} \xi \xi_{u}+\phi \phi_{u} \xi_{u}+\xi \xi_{t x}+\phi_{u} \xi_{t}+ \\
+\psi_{u} u \xi^{2}+2 \phi_{u v} u \xi^{2}+\phi_{v} \xi^{2}+\phi_{u x} \xi^{2}-\phi_{u v} \psi \xi-\phi \phi_{u u} \xi-\phi_{t u} \xi=0 \\
-u^{2} \xi^{2} \xi_{x}+\psi u \xi \xi_{x}-\phi_{v} u \xi \xi_{x}-u^{3} \xi^{2} \xi_{v}+2 \psi u^{2} \xi \xi_{v}+2 \phi_{x} u \xi \xi_{v}- \\
-\psi^{2} u \xi_{v}-\phi_{x} \psi \xi_{v}-\phi \psi u \xi_{u}-\phi \phi_{x} \xi_{u}-\psi u \xi_{t}-\phi_{x} \xi_{t}-\psi_{v} u^{2} \xi^{2}-\phi_{v v} u^{2} \xi^{2}+ \\
+\phi_{u} u^{2} \xi^{2}-2 \phi_{v x} u \xi^{2}-\phi u \xi^{2}+\psi \psi_{v} u \xi+\phi \psi_{u} u \xi+\psi_{t} u \xi-\phi_{u} \psi u \xi+ \\
+\phi_{u} \phi_{v} u \xi+\phi_{v x} \psi \xi+\phi \psi \xi+\phi \phi_{u x} \xi+\phi_{t x} \xi=0
\end{gathered}
$$

Из этих уравнений немедленно получаем

$$
\begin{aligned}
& \phi=-u^{2} \xi_{v}+\left(\psi_{v}-\xi_{x}\right) u+\psi_{x}, \\
& \xi=\alpha(x, t, v) e^{\beta(x, t, v)} .
\end{aligned}
$$

Можно выделить следующие случаи.

Случай 1. $\xi_{u} \neq 0$. Инфинитезимальные элементы можно получить, используя классический метод.

Случай 2. $\xi_{u}=0, \xi_{x} \neq 0$. Инфинитезимальные элементы можно получить, используя классический метод.

Случай 3. $\xi_{u}=0, \xi_{x}=0$. Инфинитезимальные элементы $\xi=\xi(t), \psi=\psi(x, t)$, где $\xi(t)$ и $\psi(x, t)$ должны удовлетворять уравнениям

$$
\begin{aligned}
\xi_{t} \psi+\xi^{2} \psi_{x}-\xi \psi_{t} & =0, \\
\xi_{t} \psi_{x x}-\xi \psi \psi_{x}-\xi \psi_{t x x} & =0 .
\end{aligned}
$$

Решение этих уравнений дает

$$
\xi=\delta^{\prime}(t), \quad \psi=k \delta^{\prime}(t) F(w),
$$

где $w=x+\delta(t), \delta(t)$ - произвольная дифференцируемая функция, а $F(w)$ удовлетворяет уравнению

$$
F_{w w}+\frac{k}{2} F^{2}=k_{1},
$$

где $k_{1}$ - произвольная константа. Решая характеристическое уравнение, получаем неклассическую редукцию

$$
v(x, t)=f(w)+h(z), \quad w=x+\delta(t), \quad z=x-\delta(t),
$$


где $\delta(t)$ - произвольная функция, а $F(w)=f_{w}$ и $H(z)=h_{z}$ соответственно удовлетворяют уравнениям

$$
\begin{aligned}
& F_{w w}+F^{2}=\mu_{1}, \\
& H_{z z}+H^{2}=\mu_{1},
\end{aligned}
$$

где $\mu_{1}-$ произвольная константа.

Если положить $\mu_{1}=1$, то с помощью программы Mathematica получаем решение вида

$$
f_{w}=6^{1 / 3} \mathcal{P}\left(\left(w+C_{1}\right) / 6^{1 / 3} ; 0, C_{2}\right),
$$

где $\mathcal{P}$ - функция Вейерштрасса. Проинтегрировав один раз по $w$, получаем

$$
f=-6^{2 / 3} \zeta\left(\left(w+C_{1}\right) / 6^{1 / 3} ; 0, C_{2}\right),
$$

где $\zeta$ - дзета-функция Вейерштрасса.

Решения $v=f(x+t)+h(x-t)$ и $u=f^{\prime}(x+t)+h^{\prime}(x-t)$ демонстрируют "нелинейную суперпозицию". Заметим, что такое "расщепление" решения неклассической симметрийной редукции на функцию от $x+\delta(t)$ и функцию от $x-\delta(t)$ необычно.

\section{9. ЗАКЛЮЧЕНИЕ}

В настоящей работе классический метод Ли, а также неклассический метод применены к ОУО и к интегрируемому УВ, для которого было доказано [9], что оно эквивалентно РУО. Для ОУО получено, что применение неклассического метода воспроизводит только классические симметрийные редукции. Используя простое нелинейное ОДУ, мы получили, что в случае, когда многочлен по скорости представляет собой квинтику, ОУО имеет изобилие точных решений, которые можно выразить в терминах эллиптических функций Якоби, и, следовательно, ОУО имеет изобилие решений в виде периодических волн, уединенных волн, компактонов и т.д. Для УВ применение неклассического метода к соответствующей потенциальной системе приводит к решениям, которые не возникают ни из неклассических симметрий уравнения (2), ни из потенциальных симметрий. Некоторые частные случаи решений получены с помощью классического метода Ли, предложенного в работе [10], с использованием tanh-метода и sine-cosine-метода. Некоторые решения, возникающие из неклассических редукций потенциальной системы $(12)$, демонстрируют "нелинейную суперпозицию", и, как было отмечено, такое "расщепление" решения неклассической симметрийной редукции на функцию от $x+\delta(t)$ и функцию от $x-\delta(t)$ необычно.

Благодарности. Авторы выражают благодарность за финансовую поддержку Junta de Andalucía group FQM-201 (проект P06-FQM-01448) и Ministerio de Ciencia e Innovación (проект MTM2009-11875). М.Л. Гандариас выражает благодарность В. Хереману за предоставление новой версии программы Symmgrp2009.

\section{Список литературы}

[1] Л. А. Островский, Океанология, 18:2 (1978), 181-191.

[2] Л. А. Островский, Ю. А. Степанянц, "Нелинейные волны во вращающейся жидкости", Нелинейнъе волнъ. Физика и астрофизика, ред. А. В. Гапонов-Грехов, М. И. Рабинович, Наука, М., 1993, 132-153. 
[3] R. H. J. Grimshaw, L. A. Ostrovsky, V. I. Shrira, Yu. A. Stepanyants, Surv. Geophys., 19:4 (1998), 289-338.

[4] O. A. Gilman, R. Grimshaw, Yu. A. Stepanyants, Stud. Appl. Math., 95:1 (1995), 115-126.

[5] O. A. Gilman, R. Grimshaw, Yu. A. Stepanyants, Dynam. Atmos. Oceans, 23:1-4 (1996), 403-411.

[6] A.I. Leonov, "The effect of the earth's rotation on the propagation of weak nonlinear surface and internal long oceanic waves", Fourth International Conference on Collective Phenomena, Ann. New York Acad. Sci., 373, eds. J. L. Lebowitz, Acad. Sci., New York, 1981, 150-159.

[7] В. М. Галкин, Ю. А. Степанянц, ПММ, 55:6 (1991), 1051-1055.

[8] Yu. A. Stepanyants, Chaos, Solitons and Fractals, 28:1 (2006), 193-204.

[9] V. O. Vakhnenko, E. J. Parkes, Nonlinearity, 11:6 (1998), 1457-1464.

[10] E. Yusufoğlu, A. Bekir, Chaos, Solitons and Fractals, 38:4 (2008), 1126-1133.

[11] L. Tian, J. Yin, Chaos, Solitons and Fractals, 35:5 (2008), 991-995.

[12] G. W. Bluman, J. D. Cole, J. Math. Mech., 18 (1968/69), 1025-1042.

[13] P. A. Clarkson, E. L. Mansfield, SIAM J. Appl. Math., 54:6 (1994), 1693-1719, arXiv: solv-int/9401002.

[14] N. A. Kudryashov, Chaos, Solitons and Fractals, 24:5 (2005), 1217-1231, arXiv: nlin/0406007.

[15] N. A. Kudryashov, N. B. Loguinova, Appl. Math. Comput., 205:1 (2008), 396-402.

[16] I. S. Krasil'shchik, A. M. Vinogradov, Acta Appl. Math., 2:1 (1984), 79-96.

[17] I. S. Krasil'shchik, A. M. Vinogradov, Acta Appl. Math., 15:1-2 (1989), 161-209.

[18] A. M. Vinogradov (ed.), Symmetries of Partial Differential Equations. Conservation Laws Applications - Algorithms, Kluwer, Dordrecht, 1989.

[19] И. Ш. Ахатов, Р. К. Газизов, Н. Х. Ибрагимов, “Нелокальные симметрии. Эвристический подход", Итоги науки и техн. Сер. Соврем. пробл. мат. Нов. достиж., 34, ред. Р. В. Гамкрелидзе, ВИНИТИ, М., 1989, 3-83.

[20] G. W. Bluman, G. J. Reid, S. Kumei, J. Math. Phys., 29:4 (1988), 806-811.

[21] G. W. Bluman, Z. Yan, European J. Appl. Math., 16:2 (2005), 239-261.

[22] M. L. Gandarias, M.S. Bruzon, Nonlinear Anal., 71:12 (2009), e1826-e1834.

[23] M. L. Gandarias, "New Potential symmetries", SIDE III - Symmetries and Integrability of Difference Equations, CRM Proc. Lecture Notes, 25, eds. D. Levi, O. Ragnisco, AMS, Providence, RI, 2000, 161-165.

[24] M. L. Gandarias, J. Phys. A, 29:3 (1996), 607-633. 